\title{
DIFERENCIAS EN LA CALIDAD DE VIDA DE ESTUDIANTES UNIVERSITARIOS DE DIFERENTE AÑO DE INGRESO DEL CAMPUS ANTUMAPU
}

\author{
DIFFERENCES IN UNIVERSITY STUDENTS' QUALITY \\ OF LIFE IN THE ANTUMAPUCAMPUS \\ THROUGHOUT 2005-2007
}

\author{
Samuel Durán A., Marcela Castillo A., Fernando Vio del R. \\ Instituto de Nutrición y Tecnología de los Alimentos (INTA), Universidad de Chile. Santiago, Chile.
}

\begin{abstract}
Life in university is a time of changes in education, social life, family, emotional and eating behaviors. Objective: To compare the quality of life and nutritional status in students of first year (PA) versus students of third o more (TA) years in the Antumapu Campus, University of Chile. We assessed 98 students, 52 of PA and 46 of TA, which completed a survey of quality of life, anthropometric variables and food consumption. Students of PA had a better perception of quality of life, sexual activity, relationship with their couple and wellbeing. Alcohol consumption was greater in students of TA than PA (10.6g vs $6 \mathrm{~g} p<0,05$, respectively). Women of PA had higher brachial circumference and lower waist circumference, bicipital and tricipital skin folds $(p<0.05)$. These results shows than in the perception of quality of life exist differences between gender and years of staying in the university. Students of PA presented a lower consumption of tobacco, alcohol and better nutritional status than student of TA.
\end{abstract}

Key words: Quality of life; nutritional status; food survey; anthropometry; alcohol.

Este trabajo fue recibido el 27 de Enero de 2009 y aceptado para ser publicado el 20 de Junio de 2009.

\section{INTRODUCCIÓN}

Chile se encuentra en una etapa de transición epidemiológica, que se caracteriza por presentar un aumento en la prevalencia de enfermedades crónicas no transmisibles (1), las que representan dos tercios de la mortalidad adulta del país (2). Estas enfermedades crónicas están asociadas a estilos de vida riesgosos para la salud, tales como dieta inadecuada, consumo de alcohol, tabaco e inactividad física (1).

Según la I Encuesta de Salud realizada en Chile el año 2003, la prevalencia de obesidad y sobrepeso en el grupo de 17 a 24 años, se encuentra en 9,3\% y 16,8\%, respectivamente (3). Entre los diversos factores ambientales que condicionan la obesidad, se destaca una ingesta calórica elevada y una disminución del gasto energético por actividad física, los cuales con frecuencia actúan de forma conjunta (4).

Diversos autores han descrito la vulnerabilidad nutricional de los estudiantes universitarios $(5,6)$, cuya alimentación se caracteriza por saltarse frecuentemente comidas, poco tiempo para comer, elevado costo de comidas saludables en los casinos, comer entre horas, ingesta de comida rápida e ingerir alcohol frecuentemente $(7,8)$. Si se suma a lo anterior la presión publicitaria y los regímenes de adelgazamiento mal programados (para adaptarse a los cánones de belleza imperante), estos factores pueden convertirse en factores de riesgo nutricional (6). Además, en este periodo, los estudiantes universitarios asumen la responsabilidad de su alimentación, por lo cual se convierte en una etapa crítica para el desarrollo de hábitos alimentarios, los que repercutirán en su futura salud (9).

Otro aspecto a destacar es la calidad de la alimentación, donde el aumento en el consumo de grasas juega un rol importante. En el año 2002, sobre el 27\% de la ingesta calórica total en Chile provenía de la grasa (10). En cuanto al consumo de frutas, la encuesta de Calidad de Vida del año 2000 mostró que un 53\% de las muje- 
res y sólo el $40 \%$ de los hombres comía fruta todos los días. La gran mayoría consumía sólo 1 fruta diaria (11). Además el sedentarismo en estudiantes universitarios chilenos es de un $88 \%$ (12).

En cuanto al consumo de tabaco, según la I Encuesta de Salud la prevalencia de tabaquismo en Chile en el grupo de 17 a 24 años se presenta en $61,4 \%$ en hombres y $47,4 \%$ en mujeres (11). El objetivo del presente estudio es comparar a estudiantes de primer año (PA) versus estudiantes de tercer o más años (TA) de permanencia en el Campus Antumapu de la Universidad de Chile, en relación a su valoración de la calidad de vida y estado nutricional.

\section{SUJETOS Y MÉTODOS Muestra}

La muestra estudiada corresponde a 98 estudiantes, que aceptaron voluntariamente ser parte del estudio, constituidos por 2 grupos, estudiantes de primer año o de 3 o más años de permanencia continúa en el Campus Antumapu de la Universidad de Chile (carreras de Agronomía, Ingeniería en Recursos Naturales Renovables, Ingeniería Forestal e Ingeniería en Madera). El tamaño muestral se calculó a partir de un $4 \%$ de diferencia en el porcentaje de grasa en ambos grupos, con un $\alpha$ de $5 \%$ (nivel de significancia), $\sigma=4,5$ y poder del 0,8 (13).

Los criterios de inclusión fueron, ser estudiante regular del campus de PA o TA y que haya completaron todas las evaluaciones. Criterios de exclusión: estudiantes que presentaron enfermedades crónicas (Diabetes Mellitus, Hipertensión arterial u otras), con algún régimen especial (hipocalórico u otro), que provengan de otro campus o universidad y alumnos que no completaron todos los formularios, 14 alumnos (6 de PA) y 12 (TA). A cada participante se le explicó la naturaleza y propósito del estudio, obteniendo de todos ellos un consentimiento informado.

El protocolo fue revisado y aprobado por el Comité de Ética del INTA.

\section{Procedimientos}

A cada voluntario se le aplicó una encuesta de Calidad de Vida, una evaluación antropométrica y una encuesta alimentaria (recordatorio de 24 horas modificado).

\section{Encuesta de calidad de vida}

Las preguntas de esta encuesta fueron ordenadas de acuerdo a los siguientes temas: participación en agrupaciones sociales, actividades relacionadas con el cuidado de su salud, aspectos generales de percepción de bienestar y salud, actividad física, consumo de tabaco, actividad sexual y uso de anticonceptivos.

\section{Antropometría}

Se tomaron medidas de peso $(\mathrm{kg})$, talla $(\mathrm{cm})$, perímetro de brazo $(\mathrm{cm})$, circunferencia de cintura $(\mathrm{cm})$, pliegues cutáneos (bicipital, tricipital, suprailíaco y subescapular $(\mathrm{mm})$ ) de forma estandarizada (14). Posteriormente se sumaron los 4 pliegues, se aplicó la ecuación de Durnin, para estimar la densidad corporal (15) y Siri, para estimar el porcentaje de grasa corporal (16). Para el IMC los puntos de corte fueron enflaquecido $<18,5$, normal 18,5 a 24,9 , sobrepeso 25 a 29,9 y obesidad $\geq 30 \mathrm{~kg} / \mathrm{m}^{2}$.

\section{Valoración de la ingesta dietaria}

Se utilizó un Recordatorio de Consumo de Alimentos de 24 horas modificado. Se utilizó el Programa de Evaluación de Ingesta de Alimentos del INTA, Universidad de Chile, 2006 (17). Para conocer la adecuación del aporte nutritivo de la dieta del grupo en estudio, se utilizó las recomendaciones de la OMS determinadas el 2003 (18).

\section{Análisis estadístico}

Los datos fueron procesadas en una plantilla EXCEL y en SPSS 15.0. Para evaluar normalidad de las variables continuas (edad, peso, talla. IMC) se utilizó la prueba de bondad Shapiro Wilk's; para el análisis de datos se utilizó el promedio y DE en las variables continuas y de distribución de frecuencia para las categóricas. Para las variables normales se utilizó Test t Student y para las no normales Test de Mann-Whitney U. En todos los casos se consideró significativo un valor de $\mathrm{p}<0,05$.

\section{RESULTADOS}

La muestra estudiada incluyó a 98 estudiantes voluntarios, de ambos sexos, de los cuales 52 eran PA y 46 TA. La edad, peso y talla promedio fue de $21 \pm 3$ años, $64,5 \pm 10$ kilos y $1,67 \pm 0,08$ metros, respectivamente (tabla 1). La edad presentó diferencia significativa $(\mathrm{p}<0,05)$ al comparar ambos grupos.

\section{Encuesta de calidad de vida}

Los resultados de la encuesta de calidad de vida se muestran en la figura 1 en las estudiantes de sexo femenino se observan diferencias en salud, vida general y estudios. En el caso de los estudiantes de sexo masculino, figura 2, ambos grupos tienen similitud en su buena percepción en salud, vida general, vida sexual, familia, diversión, bienestar y privacidad. Es mejor la percepción entre estudiantes de TA en estudios, pareja, condición física y dinero. 
La participación de estudiantes de sexo femenino en organizaciones comunitarias es baja: $71,4 \%$ de las estudiantes en ambos grupos no participan en ninguna organización comunitaria. La participación de los hombres en actividades comunitarias es mejor (62,5 y $55 \%$ PA y TA, respectivamente), especialmente en grupos deportivos. Llama la atención la disminución que se produce entre el PA y TA.

La prevalencia de consumo del tabaco es de 46,9\%, siendo mayor en el grupo de TA: $60,7 \%$ vs $35,7 \%$ en PA. Las estudiantes de sexo femenino de TA fuman más que las de PA, $60,7 \%$ y $35,7 \%$ respectivamente; lo mismo sucede en caso de los estudiantes de sexo masculino; $55,5 \%$ y $37,5 \%$ respectivamente.

El sedentarismo es elevado, especialmente en mujeres de TA que es de 93\%; siendo en PA de 89\%. En el caso de los estudiantes de sexo masculino, la prevalencia disminuye después de 3 años en la universidad de $84 \%$ en PA a $61 \%$ en TA.

El método anticonceptivo más utilizado es el anticonceptivo oral: $44 \%$ y $41,3 \%$, en PA y TA respectivamente y $15,2 \%$ y $13,4 \%$ utiliza condón en TA y PA respectivamente.

\section{Antropometría}

De acuerdo al IMC, el 15,3\% de los estudiantes, independiente del año de ingreso, presentó sobrepeso. La obesidad fue de un 3\%, siendo mayor el grupo de estudiantes de TA: $4,3 \%$ vs. $1,9 \%$ PA.

En la tabla 2 se observa que entre estudiantes hombres no hay diferencias significativas en las variables antropométricas. En cambio en las estudiantes de sexo

\begin{tabular}{lccccc}
\multicolumn{7}{c}{ TABLA 1 } \\
\multicolumn{7}{c}{ Características generales del grupo estudiado } \\
Variable & \multicolumn{4}{c}{ PA (n=52) } & \multicolumn{2}{c}{ TA (n=46) } & Total (n=98) \\
& Hombres & Mujeres & Hombres & Mujeres & \\
\hline Edad (años) & $19,1(2)$ & $19(2,1)$ & $23,3(2,1)$ & $23,4(2)$ & $21,1(2,0)$ \\
Peso (Kg.) & $69,4(9,4)$ & $59,1(10,4)$ & $72,6(9,7)$ & $60,8(6,8)$ & $64,5(10,5)$ \\
Talla (mt) & $1,73(0,09)$ & $1,61(0,04)$ & $1,76(0,07)$ & $1,62(0,04)$ & $1,67(0,08)$
\end{tabular}

\section{FIGURA 1}

Aspectos generales de la percepción de salud en estudiantes mujeres. (Encuesta de calidad de vida)

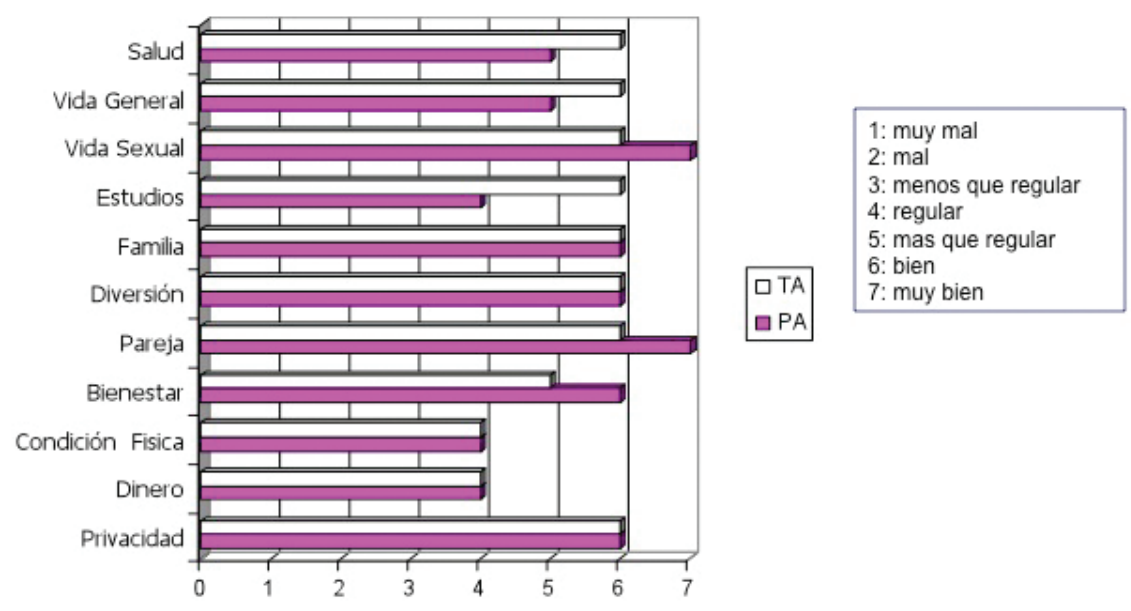


femenino de PA presentan un mayor Perímetro Muscular Braquial (PMB), menor circunferencia de cintura y pliegues bicipital y tricipital $(\mathrm{p}<0,05)$. En ambos grupos de estudiantes de sexo femenino se observa que el porcentaje de grasa está fuera del rango normal: $30,7 \%$ en PA y $32,6 \%$ en TA. En el caso de los hombres, ambos grupos muestran un porcentaje de grasa dentro de lo normal $(18,6 \%)$.

\section{Encuesta alimentaria}

El promedio del consumo de calorías fue adecuado al promedio de los requerimientos. Los hombres, inde-

\section{FIGURA 2}

Aspectos generales de la percepción de salud en estudiantes hombres.

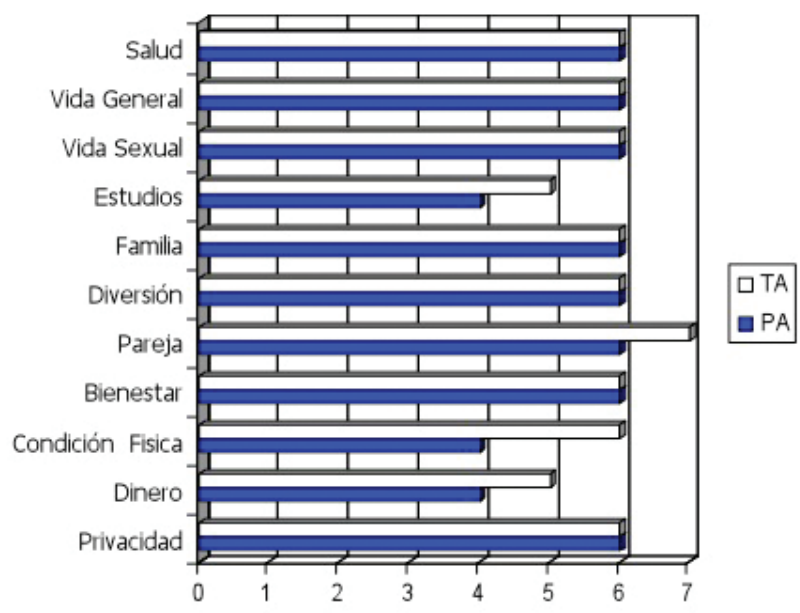

3: menos que regular

4: regular

5: mas que regular

6: bien

7: muy bien

\section{TABLA 2}

Comparación antropométrica según año de ingreso y sexo

\begin{tabular}{|c|c|c|c|c|c|c|}
\hline \multirow[t]{2}{*}{ total } & \multicolumn{2}{|c|}{ hombres } & \multicolumn{4}{|c|}{ mujeres } \\
\hline & PA & TA & $\mathbf{p}$ & PA & TA & $\mathbf{p}$ \\
\hline peso $(\mathrm{kg})$ & $69,4 \pm 9,4$ & $72,6 \pm 9,7$ & 0,2 & $59,1 \pm 10,4$ & $60,8 \pm 6,8$ & 0,16 \\
\hline talla (mt) & $1,73 \pm 0,09$ & $1,76 \pm 0,07$ & 0,15 & $1,61 \pm 0,04$ & $1,62 \pm 0,04$ & 0,47 \\
\hline IMC (kg/mt2) & $23 \pm 2,6$ & $23,3 \pm 2,8$ & 0,7 & $22,5 \pm 3,4$ & $23 \pm 2,8$ & 0,3 \\
\hline$\%$ grasa & $18,6 \pm 4,2$ & $18,6 \pm 3,6$ & 0,9 & $30,7 \pm 30,9$ & $32,6 \pm 4$ & 0,1 \\
\hline p. bicipital (mm) & $3,5 \pm 3$ & $3,9 \pm 3,9$ & 0,4 & $7,3 \pm 4,3$ & $11,2 \pm 5,7$ & $<0,01$ \\
\hline p.tricipital (mm) & $13,1 \pm 5,2$ & $11,9 \pm 5,1$ & 0,7 & $22,3 \pm 6,7$ & $25,4 \pm 6,3$ & $<0,05$ \\
\hline p.subescapular (mm) & $14,2 \pm 7,4$ & $15,1 \pm 5,7$ & 0,5 & $14,8 \pm 5,7$ & $16,3 \pm 5,6$ & 0,3 \\
\hline p.suprailiaco (mm) & $15,1 \pm 5,7$ & $19,9 \pm 9,6$ & 0,4 & $25,3 \pm 6,1$ & $25.6 \pm 8,1$ & 0,8 \\
\hline PMB (cm) & $252 \pm 19$ & $262,8 \pm 39$ & 0,4 & $207,7 \pm 20,5$ & $198 \pm 21,2$ & $<0,05$ \\
\hline Cir. Cintura $(\mathrm{cm})$ & $81,2 \pm 7,6$ & $84 \pm 7,8$ & 0,2 & $73,2 \pm 9,2$ & $77 \pm 7,9$ & $<0,05$ \\
\hline
\end{tabular}


pendiente del año de ingreso consumían significativamente más calorías que las mujeres $(\mathrm{p}<0,05)$.

Al comparar por año de ingreso e igual sexo, no existen diferencias significativas en ambos grupos, siendo superior el consumo calórico en estudiantes hombres de TA. En el caso de las mujeres, el consumo calórico mayor se observa en mujeres de PA.

El consumo de proteínas fue significativamente menor en mujeres de PA y TA con respecto a los hombres, independiente del año de ingreso $(\mathrm{p}<0,05 \mathrm{y} \mathrm{p}<0,001)$, respectivamente, no existiendo diferencias al comparar por igual sexo. En el caso de los carbohidratos y lípidos, las diferencias se observan entre hombres y mujeres, independiente al año de ingreso, siendo la ingesta significativamente superior en ambos grupos de hombres $(\mathrm{p}<0,05)$. Al comparar por igual sexo y distinto año de ingreso, sólo hay diferencias significativas entre las mujeres: las mujeres de TA consumen menos grasa que las de PA $(\mathrm{p}<0,05)$.

Al comparar el consumo de grasa por sexo y diferente año de ingreso, hay un mayor consumo de grasas en los hombres de TA que en hombres de PA $(\mathrm{p}<0,05)$.

En el caso de las vitaminas, en los hombres de ambos grupos la ingesta promedio de las vitaminas B3, B6, B12 y el mineral calcio, no alcanzaron las recomendaciones. En las mujeres, la ingesta promedio de las vitaminas B3, B6 y B12 no alcanzaron la recomendación en ambos grupos. El consumo de las vitaminas B2 y C fue insuficiente en mujeres de 3 o más años. En los minerales, la ingesta de calcio y hierro fue insuficiente en ambos grupos, siendo la recomendación de calcio para el grupo etario de 11 a 24 años, es de 1200 a 1500 mg/día (19).

El consumo de alcohol era significativamente mayor en alumnos de TA, con 10,6 g vs $6 \mathrm{~g}$ de alcohol en las estudiantes de PA $(\mathrm{p}<0,05)$.

El consumo de frutas y verduras es bajo: solo los estudiantes hombres de PA consumen más de $200 \mathrm{~g}$ al día (226,6 g/día), poco más del 50\% de los 400 g/día recomendados de frutas y verduras (18).

Al comparar por sexo y año de ingreso, solo hubo una diferencia significativa $(p<0,05)$ en el mayor consumo de frutas en los hombres de PA comparado con mujeres de TA. La ingesta de frutas en hombres de PA es de 81,6 g/día vs 58,9 g/día en TA; en el caso de las mujeres es de 64,1 g/día en PA y 40,8 g/día en TA. La ingesta de verduras en hombres de PA es de $145 \mathrm{~g} /$ día vs 135 g/día en TA; en las mujeres es de133 g/día en PA vs. $96 \mathrm{~g} /$ día en TA.

Respecto al número de comidas/día y la ingesta de frutas, figura 3 los estudiantes que comían 4 comidas diarias (desayuno, almuerzo, once y cena) la ingesta de frutas era significativamente mayor $(\mathrm{p}<0,05)(100 \mathrm{~g}$ vs. $58 \mathrm{~g}$ ) de quienes comían solo 3 comidas (desayuno, almuerzo y once). Al comparar el número de comidas e ingesta de verduras, se observa un consumo significativamente mayor $(\mathrm{p}<0,001)$ en los estudiantes que consumen 4 comidas vs quienes solo comen 3: $206 \mathrm{~g}$ vs. $96 \mathrm{~g}$ respectivamente.

\section{FIGURA 3}

Comparación entre consumo de 3 o 4 comidas e ingesta de frutas y verduras en gramos.

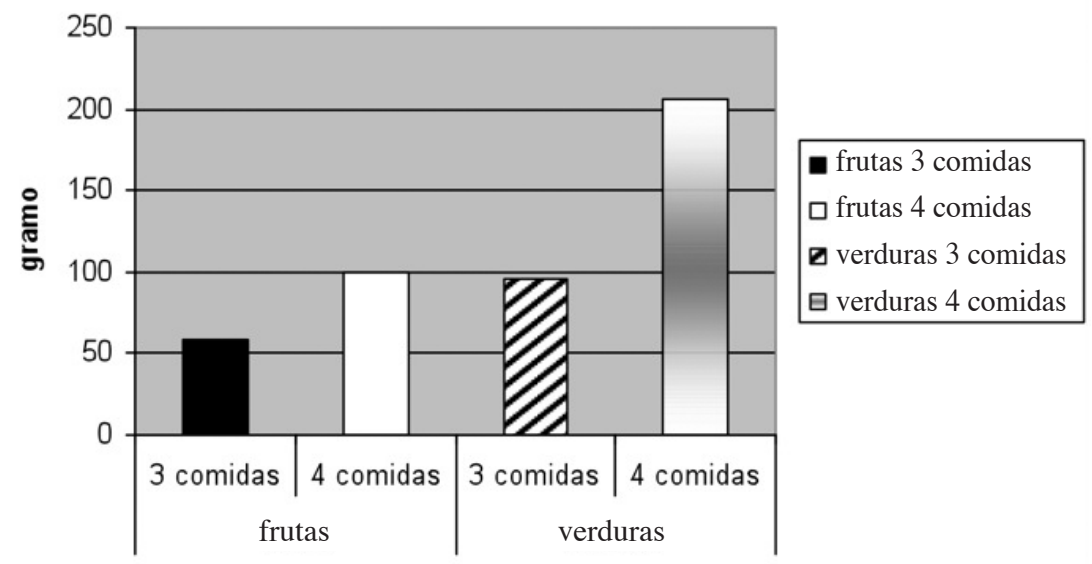

Resultados en media, diferencia significativa * p $<0,05 * *$ p $<0,05$, comparación se utilizó T-student 


\section{DISCUSIÓN}

\section{Encuesta de calidad de vida}

Al comparar los resultados sobre aspectos generales de la percepción de salud entre los estudiantes del Campus Antumapu y la II Encuesta de Calidad de Vida (20), para igual grupo etario, los estudiantes del Campus Antumapu, presentan una mejor calidad de vida en casi todos los aspectos, a excepción de dinero y estudios.

Con respecto al consumo de tabaco, al comparar nuestros resultados con los del estudio del CONACE 2004 (21) (jóvenes entre 19 y 25 años), la prevalencia del estudio CONACE fue de 59,7\% en hombres y $55 \%$ en mujeres. En el presente estudio, la prevalencia en el consumo de tabaco de los estudiantes de PA era en hombres $37,5 \%$ y $35,7 \%$ en mujeres, inferior a lo reportado por el CONACE. En cambio el consumo de tabaco en estudiantes de TA era de 55,5\% en hombres y $60,7 \%$ en mujeres, valores cercanos a los reportados por CONACE (22) en el caso de los hombres y superiores en $5 \%$ en las mujeres. Al comparar los resultados con estudios en universitarios de diferentes países (23-25), se observa la elevada prevalencia de consumo de tabaco entre los estudiantes evaluados en este estudio.

Entre los resultados destaca el aumento del consumo de tabaco en mujeres, el cual se ha asociado a cambios culturales producidos por el desarrollo económico; a medida que los países adquieren un a estructura similar a la de los países desarrollados, las diferencias en el consumo de tabaco por sexo disminuye (26).

La inactividad física es de $84 \%$ y $61 \%$ en estudiantes hombres de PA y TA respectivamente, tendencia que se repite en otro estudio chileno (12), resultado inferior a la I Encuesta Nacional de Salud (3), que muestra un $77 \%$. Al parecer, a medida que aumentan los años de universidad, disminuye la prevalencia de sedentarismo en los hombres por su participación en actividades deportivas, lo que no ocurre con las estudiantes mujeres, en las que aumenta la prevalencia de sedentarismo de un 89 a un $93 \%$.

La utilización de métodos anticonceptivos fue generalizada en los estudiantes, siendo el anticonceptivo oral el más utilizado en las mujeres y el condón es el más utilizado en los hombres. Aunque los embarazos y las enfermedades de transmisión sexual se concentran en las edades anteriores, el embarazo en la etapa universitaria puede llevar a la interrupción de la carrera o al egreso a mayor edad (27). Estudios en universitarios han encontrado un bajo conocimiento sobre métodos anticonceptivos en estudiantes y la mitad de los embarazos que ocurren en este periodo no son planificados (28).

\section{Antropometría}

La prevalencia de sobrepeso y obesidad (18\% en muestra total) es menor a la observada en la I Encuesta de Salud Chile 2003 (10) (26,1\%) y en universitarios chilenos (12) y estadounidenses (29), es similar a la obtenida por otros autores $(8,30,31)$ y superior a estudios de estudiantes chinos (13), croatas (32) y españoles (33).

La prevalencia de obesidad es mayor en los estudiantes de TA. El criterio de normalidad de acuerdo a porcentaje de grasa corporal es de $12-20 \%$ en hombres y $20-30 \%$ en mujeres. En el presente estudio, los estudiantes hombres tenían un 18,6\% y las mujeres 30,7\% y $32,6 \%$ en PA y TA respectivamente. En el caso de las estudiantes mujeres los resultados están clasificados como límite de normalidad (31-33\%) (34).

La circunferencia de cintura es mayor entre los estudiantes de TA, independiente del sexo. Lo preocupante es que el promedio de cintura de las mujeres de TA es de $77 \mathrm{~cm}$, a $3 \mathrm{~cm}$ del límite de normalidad (34).

El promedio en el PMB de los hombres, independiente del año de ingreso, y las mujeres de TA, se encuentre entre los percentiles 10 y 25 según Frisancho (35); en el caso de las mujeres de PA están en el percentil 50 (35).

\section{Encuesta alimentaria}

El estudio de ingesta alimentaria en estudiantes presenta dificultades por problemas de la estimación del tamaño de las porciones, especialmente en estudiantes hombres, además de la subestimación en el consumo de alcohol entre los estudiantes porque informan menos de lo que realmente consumen (subreporte).

El consumo energético de los 2 grupos está dentro de los rangos esperados para su edad y actividad física (36). El aporte energético derivado de los macronutrientes estaba dentro de las recomendaciones (18):13,4\% de kcal. provenientes de proteínas, $28 \%$ de kcal. de lípidos y $58,6 \%$ de carbohidratos. En el caso de los lípidos presenta un valor muy inferior al de estudios españoles, donde superan el 40\% (33,37).

La distribución del consumo de diferentes tipos de grasa también se asemejó a las recomendaciones actuales de 1:1:1 de grasas saturadas, monoinsaturadas y poliinsaturadas respectivamente. El mayor consumo de grasas poliinsaturadas coincide con otros estudios chilenos $(38,39)$.

Con respecto a la adecuación de minerales, un problema frecuente que se presenta en grupos de adultos jóvenes en nuestro país y en el mundo, es lo inadecuado que resultan las ingestas de algunos nutrientes en particular, el más habitual es el calcio, mineral cuya ingesta es baja no sólo en adultos jóvenes, sino que en 
la mayoría de los grupos etarios y que está directamente implicado en el desarrollo de la masa ósea. Este mineral se encuentra principalmente en los productos lácteos, los que son de bajo consumo en los adultos de nuestro país y de otros países $(40,41)$. La nueva recomendación de ingesta de calcio es de $1000 \mathrm{mg} /$ día (18). Solo el 19\% de los estudiantes sobrepasaba la recomendación, en cambio el 20,4\% no llegaba al 50\% de lo recomendado. Se encontró además una baja ingesta de hierro en las estudiantes mujeres, las cuales presentaron un menor consumo en alimentos de origen animal que es su principal fuente (18).

Con respecto al ácido fólico las encuestas alimentarias, que fueron realizadas después de la fortificación de la harina de panificación con ácido fólico, muestran que el 26,6\% de las mujeres presentó un consumo inferior al requerimiento (400 $\mu \mathrm{g}$ de ácido fólico) (18). Las estudiantes que tienen una ingesta baja de pan presentan un bajo aporte de ácido fólico en la dieta $(\mathrm{p}<0,05$ correlación 0,93 ).

Existe también un bajo consumo de fibra dietética, inferior a los 25-30 g recomendados. El 100\% de los alumnos no llegó a la recomendación, observándose un bajo consumo de leguminosas, alimentos integrales, frutas y verduras, principales fuentes de fibra dietética (42).

La prevalencia del consumo de alcohol es elevada, especialmente en los hombres: $88,3 \%$ y $77,7 \%$ en TA y PA respectivamente. En cambio, en las mujeres, el consumo es mayor en TA con $71,4 \%$ vs $50 \%$ en PA. Estos resultados son concordantes con el Informe sobre uso de drogas en estudiantes de la educación superior (43), el cual muestra que la prevalencia de consumo de alcohol entre estudiantes hombres y mujeres es de 80,9\% y $64,5 \%$ respectivamente, siendo mayor el consumo de alcohol en el nivel socioeconómico alto (44).

Con relación al consumo de frutas y verduras, sólo un 9,1\% llegó a la recomendación de 400 g/día. El mayor consumo se observó en los estudiantes varones y en quienes realizan 4 comidas en vez de 3 al día: al parecer los estudiantes que cenan incorporan de forma importante frutas y verduras. Sería interesante investigar este tema además de incorporar esta tendencia en las recomendaciones nutricionales. Otros autores han mostrado, en muestras con características similares, un bajo consumo de frutas y verduras en estudiantes universitarios (8).

El 72,5\% de los estudiantes evaluados consumían 3 comidas diarias, el 27,5\% restante 4 comidas. En este estudio no se observó un consumo inferior a 2 comidas; estudios en estudiantes universitarios japoneses muestra que el $81 \%$ come 3 comidas y en estudiantes coreanos el
$59,8 \%$ come solo 2 comidas al día (45). La mayoría de los alumnos ingerían alimentos a media mañana o tarde como snacks (papas fritas, ramitas), galletas, completos y bebidas, alimentos de alta densidad energética, ricos en grasa saturada, azúcar y sal, que contribuyen a la ingesta calórica total de forma importante. Con respecto al desayuno nuestro estudio muestra que el $88 \%$ de los estudiantes toman desayuno, resultado mayor comparado con los estudiantes japoneses $79 \%$ y coreanos $32,6 \%$ (45). El saltarse el desayuno se ha asociado con un peor estado nutricional y un mayor riesgo de enfermedad cardiovascular (46)

\section{Recomendaciones}

Este estudio indica lo importante que es realizar actividades de prevención del consumo de tabaco en estudiantes de primer año y la necesidad de hacer tratamiento para disminuir el consumo del tabaco en estudiantes de TA. En el control del tabaquismo se reconoce que la mejor estrategia es lograr que los individuos nunca se inicien en una conducta fumadora o que si la inician dejen de fumar lo más precozmente y se conviertan en ex fumadores (47).

Las acciones realizadas en promoción de la salud han sido aisladas; es necesario incrementar actividades coordinadas con todos los actores universitarios del campus, especialmente con los estudiantes de primer año, desde el inicio de la jornada académica, para así evitar el aumento en el consumo de tabaco y alcohol. Se debe además incentivar la actividad física, especialmente en estudiantes mujeres, incrementando la oferta académica de actividad física y las actividades extraprogramáticas. Asimismo, se debe promover el consumo de frutas, verduras y lácteos bajos en grasa entre los estudiantes, y que la alimentación entregada a los estudiantes en el casino del campus sea saludable y a un precio adecuado (48).

En conclusión estos resultados muestran que en la percepción de calidad de vida hay diferencias según sexo y año de ingreso. Los estudiantes de PA presentan un menor consumo de tabaco, alcohol, un estado nutricional más adecuado al compararlo con los estudiantes de TA; en hombres mejora el sedentarismo con la permanencia en el campus y lo contrario ocurre en las mujeres. Por lo tanto, es necesario realizar acciones de promoción de hábitos de vida saludables diferenciando sexo y año de ingreso, por las diferentes características encontradas en este estudio.

\section{RESUMEN}

El período universitario es una etapa de cambios educativos, sociales, familiares, alimentarios y emocionales. Objetivo: Comparar a estudiantes de primer año 
(PA) versus estudiantes de tres o más años de permanencia (TA) en el Campus Antumapu de la Universidad de Chile, en la valoración de su calidad de vida y estado nutricional. Método: Se evaluaron 98 estudiantes voluntarios, 52 de PA y 46 de TA y aplicándoles: una encuesta de calidad de vida y evaluación nutricional. Resultados: Los estudiantes de PA presentaron una mejor percepción en su vida sexual, pareja y bienestar. El consumo de alcohol era significativamente mayor en alumnos de TA $10,6 \mathrm{~g}$ vs PA $6 \mathrm{~g}(\mathrm{p}<0,05)$. Estudiantes mujeres de PA presentan un mayor Perímetro Muscular Braquial, menor circunferencia de cintura y pliegues bicipital y tricipital $(\mathrm{p}<0,05)$. Conclusión: La percepción de calidad de su vida es diferente según sexo y año de ingreso, los estudiantes de PA presentan un menor consumo de alcohol y un estado nutricional más adecuado al comparados con los estudiantes de TA.

Palabras clave: calidad de vida, estado nutricional, encuesta alimentaria, antropometría y alcohol.

Dirigir la correspondencia a:

Profesor

Samuel Durán A.

Laboratorio del Sueño

INTA, Universidad de Chile

Email: sduran@inta.cl

Fono: 02-9781447

Fax : 02-2214030

\section{BIBLIOGRAFÍA}

1. Concha M, Aguilera X, Guerrero A, González C, Salas J: Situación de Salud en Chile 1996. Santiago: Ministerio de Salud 1996

2. Salinas J. Vio F. Promoción de la Salud en Chile. Rev Chil Nutr 2002; 29:164-73

3. I Encuesta de Salud, Ministerio de Salud, Chile 2003. http://www.bcn.cl/carpeta_temas/temas_ portada.2006-09-25.0806013222/documentos-pdfsobre-obesidad/VIGIA20.pdf

4. Uauy R. Atalah E, Kain J. The nutritional transition: new nutritional Influences on child growth. In: Martorell R, Haschke F, editors Nutrition and growth. Williams and Wilkins, Philadelphia, USA, 2001; pp 305-328

5. López-Azpiazu I, Sánchez-Villegas A, Johansson L, Petkeviciene J, Prattala R, Martínez-González MA. Disparities in food habits in Europe: systematic review of educational and occupational differences in the intake of fat. J Hum Nutr Diet 2003; 16: 349364

6. López Nomdedeu C. Influencia de la estructura social y familiar de los hábitos alimentarios. En
Tratado de Nutrición. Hernández M, Sastre A (eds). pp 1355-1365. Díaz de Santos 1999.

7. Bellisle F, Monneuse MO, Steptoe A, Wardle J. Weight concerns and eating patterns - A survey of university students in Europe. Int J Obes 1995; 19:723-730

8. Arroyo M, Rocandio AM, Ansotegui L, Pascual E, Salces I, Rebato E. Calidad de la dieta, sobrepeso y obesidad en estudiantes universitários. Nutr Hosp. 2006; 21: 673-679

9. Barić I, Ŝataliĉ Z, Lukešić Ž. Nutritive value of meals, dietary habits and nutritive status in Croatian university students according to gender. Int J Food Sci Nutr 2003;54:473-484.

10. Vio F, Albala C. Nutrition transition in Chile: case study. In: FAO. Globalization of food systems in developing countries: impact on food security and nutrition. Food and Agriculture Organization of the United Nations, Rome. FAO 2004;83: 275-284

11. Chile. Ministerio de Salud. Departamento de Epidemiología. Departamento de Promoción de la Salud. I Encuesta Nacional de calidad de Vida. Santiago, Chile 2000.

12. Mc Coll P, Amador M, Aros J, Lastra A, Pizarro C. Prevalencia de factores de riesgo de enfermedades crónicas no transmisibles en estudiantes de medicina de la Universidad de Valparaíso. Rev Chil Pediatr 2002, 73;478-482.

13. Miyatake N, Kogashiwa M, Wang D, Kira H, Yamasato T, Fujii M. The relation between visceral adipose tissue acumulation and biochemical tests in university students.Acta Med. Okayama 2005;59:129-134

14. Lohoman TG, Roche AF, Martorell R (Eds). Anthropometric standardization reference manual. Champaing,IL: Human Kinetics 1988

15. Durnin JVGA, Womersley J. Body fat assessment from total body density and its estimation from skinfold thickness: measurements on 481 men and women aged from 16 to 72 years. Br J Nutr 1974; 32:77-96

16. Siri WE. Body composition from fluid spaces and density: analysis of methods. In: Techniques for Measuring Body Composition. Brozek J. Henschel A (eds). Washington DC: National Academy of Sciences. 1961; pp 223-244.

17. Programa de Evaluación de Ingesta de Alimentos, INTA, Universidad de Chile, 2006.

18. FAO/OMS. Diet, Nutrition And The Prevention Of Chronic Diseases. WHO, Geneva 2003. Technical Document. http://www.fao.org/docrep/005/ AC911E/ac911e05.htm\#bm05.6 
19. National Institute of Health. Optimal calcium intake. NIH Consensus Statement. Am J Med 1994; 12:1-32.

20. II Encuesta de Calidad de Vida y Salud Chile 2006. http://epi.minsal.cl/epi/html/sdesalud/calidaddevida2006/II\%20Encuesta\%20de $\% 20$ Calidad $\% 20$ de\%20Vida\%20y\%20Salud\%202006.pdf

21. Minuta informativa sobre el consumo de cigarrillos en Chile. Estudios Nacionales de Drogas en Población General de Chile 1994 - 2004 http:// www.google.cl/search?q=conace $+2004 \& i e=u t f-$ $8 \& o e=u t f-\& a q=t \& r l s=$ org.mozilla:es-ES:official $\&$ client $=$ firefox-a

22. Uso de drogas en estudiantes de educación superior http://www.conace.cl/inicio/pdf/Uso_drogas_estudiantes_educacion_superior_CONACE2004.pdf

23. Guillén D, Nerin I, Mas A y Crucelaegui A. Estudio de fiabilidad de una encuesta utilizada para valorar la prevalencia, los conocimientos y las actitudes sobre el tabaquismo en estudiantes de medicina. Arch Bronconeumol 2003; 39: 159-166.

24. Alegre M, Gutiérrez A, Oliván V, Vara E, MartínezGonzález MA, Aguinaga I, et al. Prevalencia del tabaquismo, conocimiento y valoración de los métodos de cesación tabáquica en estudiantes de medicina. Anales Sis San Navarra 1998; 21: 233-240.

25. Arrivillaga M, Salazar I, Correa D. Creencias sobre la salud y su relación con las prácticas de riesgo o de protección en jóvenes universitarios. Colomb Med 2003;34:186-195

26. Rojas G, Gaete J, González I, Ortega M, Figueroa A, Fritsch R, Araya R. Tabaquismo y salud mental. Rev Méd Chile 2003; 131:873-880

27. Perez M, Padilla M, Serva L, Parada H. Embarazo no planificado de estudiantes universitarias en control pre-natal. MedULA 1994;2;1-9

28. Silva P, Alvarado R, Palavecino 1, Sandoval U, Quevedo F, Girárdi G, Aranda W. Conocimientos sobre sexualidad en estudiantes universitarios. Cuad. Mcd. Soc.1988; 29:11-18

29. Lowry R, Galuska D, Fulton J, Wechsler H, Kann L y Collins J. Physical activity, food choice, and weight management goals and practices among U.S. college students. AJPM 2000;18: 18-27

30. González-Cross M, Castill MJ, Moreno L y cols. Alimentación y valoración del estado nutricional de los adolescentes españoles (estudio AVENA). Nutr Hosp 2003; 23: 15-28.

31. Martínez C, Veiga P, López de Andrés A, Cobo JM, Carbajal A. Evaluación del estado nutricional de un grupo de estudiantes universitarios mediante parámetros dietéticos y de composición corporal.
Nutr Hosp 2005; 20: 197-203.

32. Colic I, Šatalic Z, Lukesic Ž. Nutritive value of meals, dietary habits and nutritive status in Croatian university students according to gender. Int J Food Sci Nutr 2003;54: 473-484

33. Montero P, Morales E, Carvajal A. Valoración de la percepción de la imagen corporal mediante modelos anatómicos. Rev Antropol 2004;8:107-116.

34. Albala C, Arroyo P. Evaluación de la Obesidad en el adulto. Rev Chil Pediatr 2006; 77; 20-26.

35. Barrera MG, Salazar G, Gajardo H, Gattás V, Coward A. Análisis comparativo de métodos de evaluación de la composición corporal en varones sanos adultos. Rev Med Chile 1997; 125:1335-1342

36. Human Energy Requirements. Report of a Joint FAO/WHO/UNU Expert Consultation. Rome 2001 pag 35-50

37. Montero A, Úbeda N, García A. Evaluación de los hábitos alimentarios de una población de estudiantes universitarios en relación con sus conocimientos nutricionales. Nutr Hosp 2006;21:466-473

38. Atalah E, Arteaga C, Rebolledo A, Delfín S, Ramos R. Patrones alimentarios y de actividad física en escolares de la Región de Aysén. Rev Chil Pediatr 1999; 70:483-490

39. Castillo O, Rozowski J, Jadue L.Hábitos alimentarios de la población de Valparaíso: Encuesta de base CARMEN. Rev Chil Nutr 2000; 27: 56-61.

40. Atalah E, Benavides X, Avila L, Barahona S, Cárdenas R. Características alimentarias de adultos mayores de comunas pobres de la Región Metropolitana. Rev Méd Chile 1998; 126: 489-96.

41. Castillo O, Rozowski J, Jadue L.Hábitos alimentarios de la población de Valparaíso: Encuesta de base CARMEN. Rev Chil Nutr 2000; 27: 56-61.

42. Jury G, Arteaga C y Taibo M. Porciones de intercambio y composición química de los alimentos de la pirámide alimentaria chilena. Universidad de Chile. INTA. Centro de Nutrición Humana Facultad de Medicina.1999 pp 72-74.

43. Guía clínica consumo perjudicial y dependencia de alcohol y drogas en menores de 20 años. http:// www.redsalud.gov.cl/archivos/guiasges/GPCGesOHyDrogas-2007-rev26junio.pdf

44. Informe sobre uso de drogas en estudiantes de educación superior. http://www.conace.cl/inicio/ pdf/Uso_drogas_estudiantes_educacion_superior_CONACE2004.pdf

45. Sakamaki R, Amamoto R, Mochida Y, Shinfuku $\mathrm{N}$, Toyama K. A comparative study of food habits and body shape perception of university students in Japan and Korea. Nutr J 2005;4:31 
46. Sakata K, Matumara Y, Yoshimura N, Tamaki J, Hashimoto T, Oguri S, Okayama A, Yanagawa H. Relationship between skipping breakfast and cardiovascular disease risk factors in the national nutrition survey data. Nipón Koshu Eisei Zasshi 2001; 48:837-8473

47. Behn V, Sotomayor H, Cruz M, Naveas R. Mo- tivaciones para dejar de fumar en funcionarios y estudiantes universitarios ex fumadores. Rev Méd Chile 2001;129: 540-546

48. Lange I, Vio F.Guía para Universidades Saludables y otras instituciones de educación superior. Santiago: OMS/OPS / Universidad de Chile, INTA / PUC UC Saludable / Vida Chile; 2006. 